\title{
miR-125b-mediated regulation of cell proliferation through the Jagged-1/Notch signaling pathway by inhibiting BRD4 expression in psoriasis
}

\author{
MIN PAN ${ }^{1,2^{*}}$, YAO HUANG ${ }^{3 *}$, XIAOFANG ZHU ${ }^{4}$, XIANGFEI LIN ${ }^{4}$ and DAN LUO ${ }^{1}$ \\ ${ }^{1}$ Department of Dermatology, The First Affiliated Hospital of Nanjing Medical University, Nanjing, Jiangsu 210029; \\ ${ }^{2}$ Department of Dermatology, Nanjing First Hospital, Nanjing Medical University, Nanjing, Jiangsu 210006; \\ ${ }^{3}$ Institute of Sport Medicine, Affiliated Hospital of Nanjing University of TCM, Nanjing, Jiangsu 210023; \\ ${ }^{4}$ Department of Dermatology, Northern Jiangsu Province Hospital, Yangzhou, Jiangsu 225001, P.R. China
}

Received April 23, 2018; Accepted January 3, 2019

DOI: $10.3892 / \mathrm{mmr} .2019 .10187$

\begin{abstract}
Psoriasis is a chronic inflammatory disease characterized by the abnormal differentiation and hyperproliferation of epidermal keratinocytes. The aim of the present study was to investigate the mechanism by which microRNA-125b (miR-125b) inhibits the activation of the bromodomain-containing protein 4 (BRD4)/Notch signaling pathway in psoriasis. The contents of associated miRNAs in serum samples from 32 patients with psoriasis were detected by reverse transcription-quantitative polymerase chain reaction (RT-qPCR). The most significantly downregulated miRNA, miR-125b, was screened out. In experiments using $\mathrm{HaCaT}$ cells, the association between miR-125b and cell proliferation was observed using a Cell Counting Kit- 8 assay, that between miR-125b and the Notch signaling pathway was observed by western blotting and RT-qPCR, and that between miR-125b and the upstream molecule BRD4 of the Notch signaling pathway was observed by luciferase reporter assay and western blotting. The proliferation of $\mathrm{HaCaT}$ cells became apparent following miR-125b inhibition. The Jagged-1 ligand in the Notch signaling pathway was upregulated, the active intracellular domain of the Notch1 receptor was increasingly truncated, and the Notch signaling pathway was activated. Furthermore, the inhibited miR-125b contributed directly toward the upstream protein BRD4 3'-UTR of Jagged-1, ultimately activating the Notch signaling pathway with the upregulation of Jagged-1. In
\end{abstract}

Correspondence to: Dr Dan Luo, Department of Dermatology, The First Affiliated Hospital of Nanjing Medical University, 300 Guangzhou Road, Nanjing, Jiangsu 210029, P.R. China

E-mail: daniluo2005@163.com

*Contributed equally

Key words: miR-125b, Jagged-1, bromodomain-containing protein 4 , Notch signaling pathway, proliferation conclusion, the proliferation of $\mathrm{HaCaT}$ cells mediated by the Jagged-1/Notch signaling pathway was decreased with the miR-125b-mediated inhibition of BRD4 expression. Therefore, miR-125b may be a biomarker and potential therapeutic target for psoriasis treatment.

\section{Introduction}

Psoriasis is a common chronic inflammatory skin disease and affects $0.1-3 \%$ of the world population (1). This disease is stubborn, recurrent and difficult to cure, and seriously affects the quality of life of patients (2). The immunological mechanism of the onset of psoriasis is of great concern. Psoriasis is a chronic inflammatory condition, where psoriatic lesions are formed through the interactions between keratinocytes and immune cells. Abnormal human keratinocyte proliferation is an important feature of psoriasis. The hyperproliferation and abnormal differentiation of keratinocytes comprise the typical histopathological basis for the formation of epidermal hypertrophy and parakeratosis (3).

Typical Notch signaling pathways have been introduced. Mammals possess four types of Notch receptors (Notch-1, Notch-2, Notch-3 and Notch-4) and five types of ligands (Jagged-1/-2 and Delta-like 1, 3 and 4). The Notch signaling pathway serve an important role in cell differentiation, proliferation and apoptosis (4), and it is correlated with inflammation. This pathway is active in numerous inflammatory diseases, including psoriasis, rheumatoid arthritis and systemic lupus erythematosus $(5,6)$. Jagged-1 expression is markedly upregulated in patients with psoriatic lesions as the binding of the Jagged-1 protein to the Notch receptor activates the Notch signaling pathway and promotes epidermal hyperplasia (7). Jagged-1 protein is a transmembrane protein on the cell surface and is widely present in different cells of the peripheral immune system. In particular, this protein serves important roles in the activation, proliferation and differentiation of peripheral $\mathrm{CD}^{+} \mathrm{T}$ cells (8). Therefore, the Jagged-1 protein-mediated Notch signaling pathway may be involved in the hyperproliferation of psoriatic keratinocytes and the abnormal activation of the immune system (9). 
MicroRNAs (miRNAs) are single-stranded, non-coding, short RNA molecules. They are gene expression regulators that trigger target mRNA degradation and inhibit translation, thereby regulating biological processes, including cell proliferation, differentiation, apoptosis and tumorigenesis. Therefore, miRNAs serve critical roles in epidermal disorders, including psoriasis. miR-31 was observed in lesional and non-lesional skin of patients with psoriasis and was triggered by activating NF- $\mathrm{KB}$ to promote keratinocyte hyperproliferation in psoriasis $(10,11)$. Additionally, miR-21 inhibits the apoptosis of activated $\mathrm{T}$ cells in psoriasis (12). Furthermore, miR-125b is becoming a popular area of molecular research. It is highly expressed in skin stem cells and in an inducible mouse system, and is associated with stem cell differentiation and inhibition (13). By identifying the potential role of miR-125b in psoriasis pathogenesis, researchers have noted that the most notably downregulated miRNA in psoriatic skin is miR-125b. As indicated by in situ hybridization results, the cell type mainly involved in the downregulation of miR-125b in psoriatic lesions is the keratinocyte, and a decrease or loss of miR-125b in psoriatic skin may cause abnormality in the hyperproliferation and differentiation of such cells $(14,15)$.

When miRNA was detected in the sera of 32 patients with psoriasis in previous studies, the expression of miR-125b was markedly decreased. Bromodomain-containing protein 4 (BRD4), a member of the Bromodomain and extra-terminal domain (BET) family, activates the NF- $\mathrm{KB}$ signaling pathway and is associated with inflammation and cancer $(16,17)$. The association between miR-125b and the BRD4/Notch signaling pathway with regard to psoriasis has been poorly reported. The present study aimed to investigate the mechanism of action between miR-125b and the BRD4/Notch signaling pathway, and to investigate novel targets and ideas for treating psoriasis.

\section{Materials and methods}

The present study was approved by the Ethics Committee of the Northern Jiangsu Province Hospital (Jiangsu, China).

Human serum. A total of 32 subjects (18 males and 14 females; aged 18-39 years; mean age, $27.28 \pm 6.33$ years) were enrolled in the present study. The sera of 10 healthy volunteers $(5$ males and 5 females; aged 20-35 years; mean age, 28.1 \pm 5.53 years) for the control group were provided by the Department of Dermatology of Northern Jiangsu Province Hospital (Jiangsu, China). The sera were sampled, centrifuged $(1,000 \mathrm{x} \mathrm{g}$ for $10 \mathrm{~min}$ at $4^{\circ} \mathrm{C}$ ) and stored at $-70^{\circ} \mathrm{C}$.

Cell culture. The HaCaT and 293T cells were purchased from Chinese Academy of Sciences Cell Bank (shanghai, China). The cells were cultured with RPMI-1640 medium (Gibco; Thermo Fisher Scientific, Inc., Waltham, MA, USA), supplemented with $10 \%$ fetal bovine serum (Hyclone; GE Healthcare Life Sciences, Logan, UT, USA) and $1 \%$ streptomycin-penicillin (Gibco; Thermo Fisher Scientific, Inc.).

Cell Counting Kit-8 (CCK-8). Cells were inoculated at $1 \times 10^{5} / \mathrm{ml}$ in 96 -well culture plates $(\sim 5,000$ cells in $100 \mu \mathrm{l}$ medium per well), $100 \mu \mathrm{l}$ serum-free medium was then added, and cells were starved for $6 \mathrm{~h}$. The medium was replaced with complete medium containing different concentrations of DAPT (5, 10 and $20 \mathrm{nmol} / \mathrm{l}$; cat. no. HY-13027; MedChemExpress LLC, Monmouth Junction, NJ, USA). Controls included three wells without cells, and three wells with saline-treated cells. The complete medium was replaced with $100 \mu 1$ serum-free medium containing $10 \%$ CCK- 8 dye after 1, 2 and 3 days, and cells were cultured for an additional $1 \mathrm{~h}$. The optical absorbance at $450 \mathrm{~nm}$ for each sample was measured using an absorbance microplate reader (ELx800 Absorbance Microplate Reader; BioTek Instruments, Inc., Winooski, VT, USA). Survival of neurons in the saline control group was defined as $100 \%$ and the results are expressed as percentage relative to the control values.

RNA extraction and reverse transcription-quantitative polymerase chain reaction ( $R T-q P C R)$. Total RNA was extracted using an RNA extraction kit (RNA Quickly Extraction Kit, BioTeke Corporation, Beijing, China). Following quantification, RNA was reverse transcribed into the first strand of cDNA using an an iScript ${ }^{\mathrm{TM}}$ cDNA kit (Bio-Rad Laboratories, Inc., Hercules, CA, USA) at $37^{\circ} \mathrm{C}$ for $15 \mathrm{~min}$ and $85^{\circ} \mathrm{C}$ for $5 \mathrm{sec}$, prior to storage at $4^{\circ} \mathrm{C}$. Real-Time PCR Detection was performed using iTaq ${ }^{\mathrm{TM}}$ SYBR-Green (Applied Biosystems; Thermo Fisher Scientific, Inc.). The samples were treated with recombinant DNase I (DNA-free DNA removal kit; Ambion; Thermo Fisher Scientific, Inc.) to remove possible DNA contamination. $\beta$-actin was used as an internal control. The primer sequences used during the present study are presented in Tables I and II. The thermocycling conditions were as follows: Initial pre-denaturation at $95^{\circ} \mathrm{C}$ for $1 \mathrm{~min}$, denaturation for $15 \mathrm{sec}$, annealing at $55-65^{\circ} \mathrm{C}$ for $20 \mathrm{sec}$ and extension at $72^{\circ} \mathrm{C}$ for $30 \mathrm{sec}$. A total of 50 cycles were performed. Based on the $\mathrm{Cq}$ value and relative standard curve of the PCR product, the amount of RNA template contained in each specimen was determined and compared with the amount of $\beta$-actin. The specific value of the amount of RNA template to that of $\beta$-actin was adopted as the final statistical value. The results were processed using the $2^{-\Delta \Delta \mathrm{Cq}}$ method (18).

Western blot analysis. Cells were placed on ice immediately following treatment and washed with ice-cold Hank's balanced salt solution (HBSS). All the wash buffers and final resuspension buffer included $1 \mathrm{X}$ protease inhibitor cocktail (Pierce; Thermo Fisher Scientific, Inc.), $\mathrm{NaF}(5 \mathrm{mM})$ and $\mathrm{Na}_{3} \mathrm{VO}_{4}$ $(200 \mathrm{mM})$. The protein concentration of the lysate was measured using a Bicinchoninic Acid Protein Assay kit (Thermo Fisher Scientific, Inc.). Equal amounts ( $30 \mu \mathrm{g}$ ) of total protein were subjected to $8-12 \%$ SDS-PAGE separation and transferred via electroblotting to nitrocellulose membranes (Bio-Rad Laboratories, Inc.). The membranes were blocked in non-fat dry milk solution (5\%, room temperature, $2 \mathrm{~h}$ ) and incubated overnight at $4{ }^{\circ} \mathrm{C}$ with primary antibodies (all from Abcam, Cambridge UK) against Hes1 (1:1,000; cat. no. ab71559), Hey1 (1:500; cat. no. ab22614), Hey2 (1:500; cat. no. ab25404), Jagged-1 (1:1,000; cat. no. ab109536), BRD4 (1:1,000; cat. no. ab75898), $\beta$-tubulin (1:1,000; cat. no. ab179513), GAPDH (1:1,000; cat. no. ab181602) and Notch1 intracellular domain 1 (NICD1; 1:1,000; cat. no. ab83232). Membranes were then incubated with horseradish peroxidase-conjugated anti-mouse immunoglobulin G (IgG; 1:5,000; cat. no. G-21040, Thermo 
Table I. miRNA primer sequences used in reverse transcription-quantitative polymerase chain reaction.

\begin{tabular}{|c|c|}
\hline Gene & Primer sequence \\
\hline \multirow[t]{2}{*}{$\beta$-actin } & Forward: 5'-CATGTACGTTGCTATCCAGGC-3' \\
\hline & Reverse: 5'-CTCCTTAATGTCACGCACGAT-3' \\
\hline \multirow[t]{2}{*}{ miRNA-125b } & Forward:5'-CTTGCCAGAAACGTCAATGGA-3' \\
\hline & Reverse: 5'-GTGCAACTACGTCATAGCCTG-3' \\
\hline \multirow[t]{2}{*}{ miRNA-200c } & Forward:5'-CTTGCCAGAAACGTCAATGGA-3' \\
\hline & Reverse: 5'-GTGCAACTACGTCATAGCCTG-3' \\
\hline \multirow[t]{2}{*}{ miRNA-200b } & Forward: 5'-ACTGGTGGGTATGGGCATTG-3' \\
\hline & Reverse: 5'-GCGCAGATGAACACGAACAG-3' \\
\hline \multirow[t]{2}{*}{ miRNA-19b } & Forward: 5'-GAGCCTGGGTTCGACGATG-3' \\
\hline & Reverse: 5'-CCTGCTCTCGCTTATCTCCA-3' \\
\hline \multirow[t]{2}{*}{ miRNA-152 } & Forward: 5'-ACAGGCAGACACTAACGTCC-3' \\
\hline & Reverse: 5'-CTGGGGTAGGATGCGAGGA-3' \\
\hline \multirow[t]{2}{*}{ miRNA-296-5p } & Forward: 5'-GAAGGGCCCCCCCTCA-3' \\
\hline & Reverse: 5'-GTGCGTGTCGTGGAGTCG-3' \\
\hline \multirow[t]{2}{*}{ miRNA-30d } & Forward: 5'-CCTGTTGGTGCACTTCCTAC-3' \\
\hline & Reverse: 5'-TGCAGTAGTTCTCCAGCTGC-3' \\
\hline \multirow[t]{2}{*}{ miRNA-611 } & Forward: 5'-AGGCGAGGACCCCT-3' \\
\hline & Reverse: 5'-GTCCAGTTTTGTCAG-3' \\
\hline \multirow[t]{2}{*}{ miRNA-419-3p } & Forward: 5'-GCACTGGATACGACGTAGAA-3' \\
\hline & Reverse: 5'-GCCCCTTATGCAAGATTCCC-3' \\
\hline
\end{tabular}

miRNA, microRNA.

Table II. mRNA primer sequences used in reverse transcriptionquantitative polymerase chain reaction.

Gene $\quad$ Primer sequence

$\beta$-actin Forward: 5'-CATGTACGTTGCTATCCAGGC-3' Reverse: 5'-CTCCTTAATGTCACGCACGAT-3'

Hes1 Forward: 5'-TCAACACGACACCGGATAAAC-3' Reverse: 5'-CTCCTTAATGTCACGCACGAT-3'

Hey1 Forward: 5'-GTTCGGCTCTAGGTTCCATGT-3' Reverse: 5'-CGTCGGCGCTTCTCAATTATTC-3'

Hey2 Forward: 5'-CCTAACAGAAGTTGCGCGGTA-3' Reverse: 5'-GAGGCGACAAGGGGTTGAC-3'

Jagged-1 Forward: 5'-GTCCATGCAGAACGTGAACG-3' Reverse: 5'-GCGGGACTGATACTCCTTGA-3'

Jagged-2 Forward: 5'-TGGGCGGCAACTCCTTCTA-3' Reverse: 5'-GCCTCCACGATGAGGGTAAA-3'

DLL1 Forward: 5'-GATTCTCCTGATGACCTCGCA-3' Reverse: 5'-TCCGTAGTAGTGTTCGTCACA-3'

BRD4 Forward: 5'-GAGCTACCCACAGAAGAAACC-3' Reverse: 5'-GAGTCGATGCTTGAGTTGTGTT-3'

BRD4, bromodomain-containing protein 4; DLL1, $\delta$-like canonical Notch ligand 1.

Fisher Scientific, Inc.) and anti-rabbit IgG antibodies (1:5,000; cat. no. 31460, Thermo Fisher Scientific, Inc.) for $2 \mathrm{~h}$ at room temperature. Afterwards, the membranes were developed using the enhanced chemiluminescence substrate LumiGLO (EMD Millipore, Billerica, MA, USA) and exposed to X-ray film. The bands were analyzed using Gel-Pro Analyzer 4.0 (Media Cybernetics, Inc., Rockville, MD, USA).

Bioinformatics analysis. The target genes of miR-125b were analyzed using the target prediction tool TargetScan version 7.1 (http://www.targetscan.org/vert_71/) and miRanda version 3.3a (http://www.microrna.org/microrna/home.do).

Transient transfection. The cells (50\% confluence) were transfected with miR-125b mimic (50, 100 and $150 \mathrm{nmol} / \mathrm{l})$, inhibitor $(25,50$ and $100 \mathrm{nmol} / \mathrm{l})$ or BRD4 small interfering RNA (siRNA; 50 nmol/l; all Guangzhou RiboBio Co., Ltd., Guangzhou, China), according to the manufacturer's protocols. For experiments where only one concentration was used, cells were transfected with $50 \mathrm{nmol} / 1 \mathrm{mimic} /$ inhibitor/siRNA. miR-negative control (NC; $50 \mathrm{nmol} / \mathrm{l})$, inhibitor NC (50 nmol/l) and si-NC (50 nmol/l) were used as respective transfection controls. The transfection reagent used was Lipofectamine ${ }^{\circledR} 2000$ (Thermo Fisher Scientific, Inc.). The sequence of BRD4 siRNA was 5'-GACUAGAAACUUCCCAAAUGUCUUUCAAGA GAAGACAUUUGGGAAGUUUCUAGUC-3'. The sequence of miR-125b mimic was sense, 5'-UCACAAGUUAGGGUC UCAGGGA-3' and antisense, 3'-AGUGUUCAAUCCCAGAGU CCCU-5'. The sequence of miR-125b inhibitor was 5'-UCACAA GUUAGGGUCUCAGGGA-3'. The sequence of miR-NC was 5'-GGCUGCCUACUUAGCUUGAGAGUG-3'. The sequence of inhibitor NC was 5'-ACGGGUGUGACCACUCCAGGC 


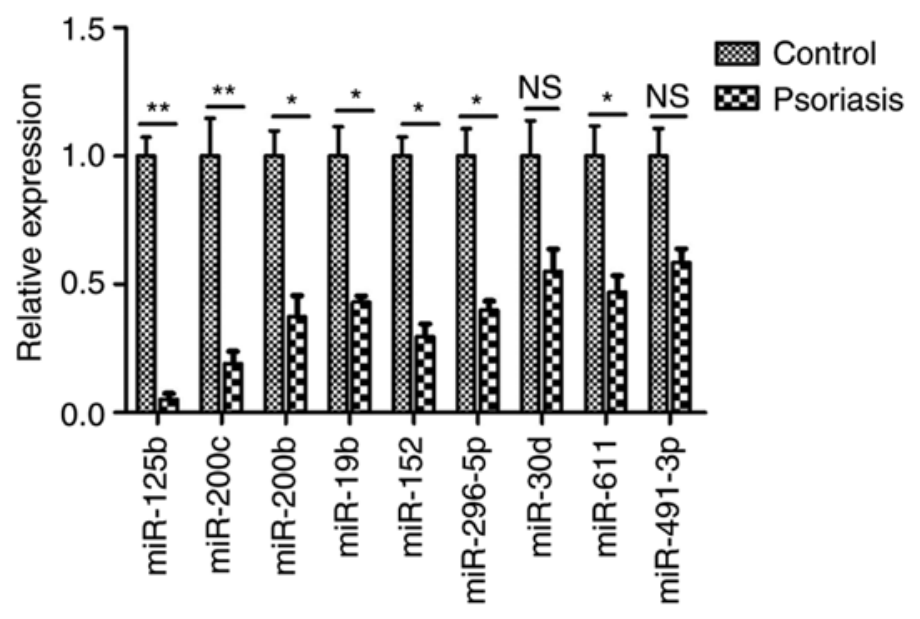

Figure 1. Comparisons of the expression levels of different miRNAs in patients with psoriasis and healthy controls. Of the different miRNAs, miR-125b was most notably downregulated. ${ }^{*} \mathrm{P}<0.05 ;{ }^{* *} \mathrm{P}<0.01$; NS, not significant. miRNA/miR, microRNA.

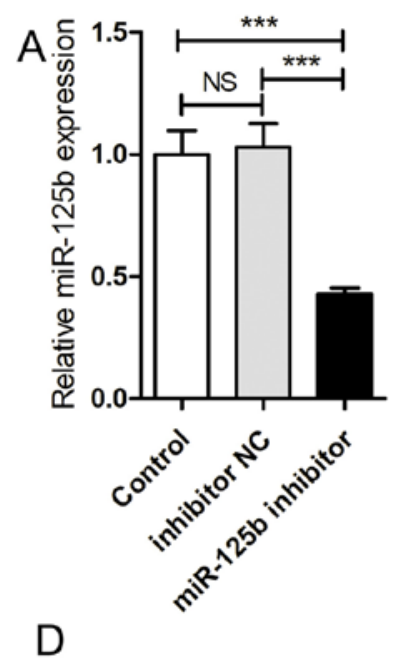

B
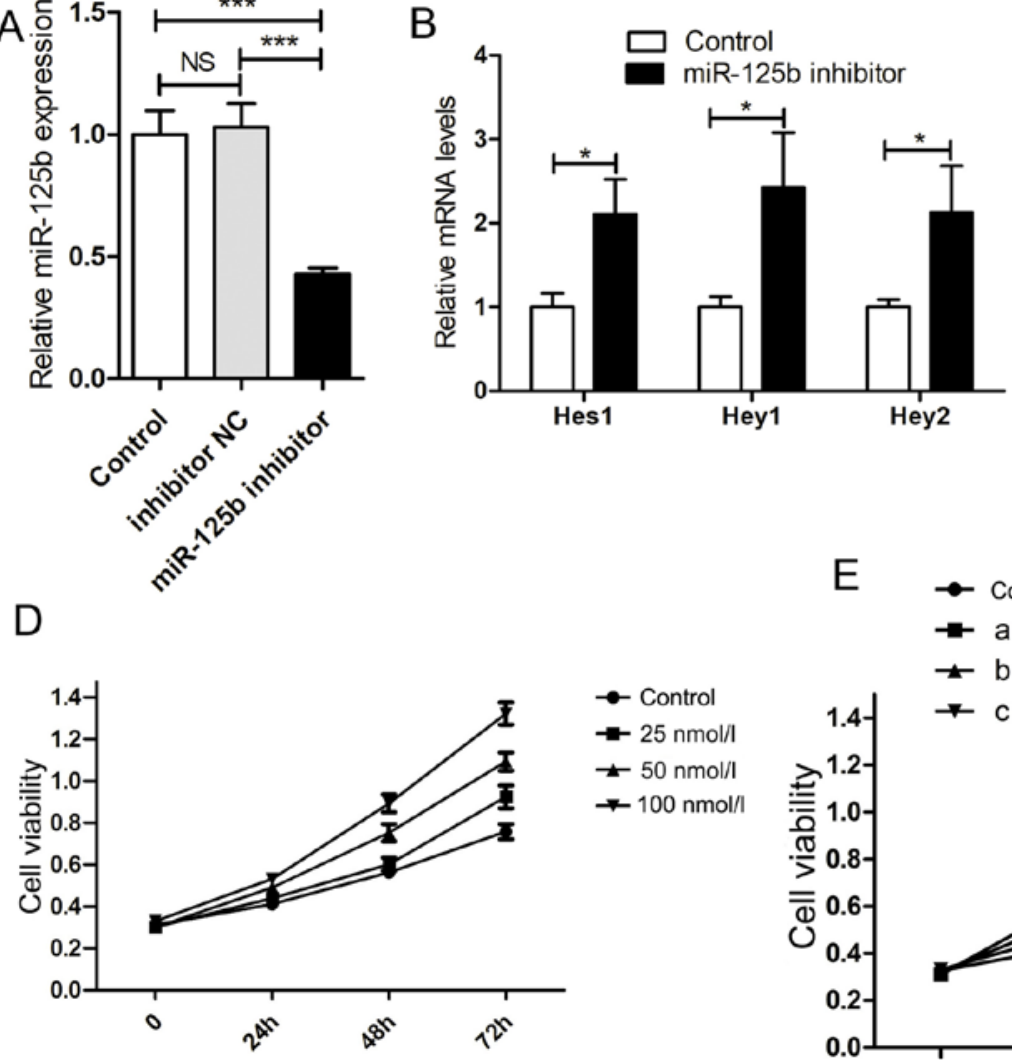

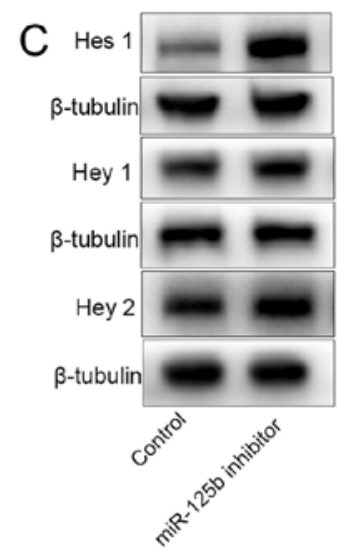

Inhibitor(nmol/l) DAPT $(\mu \mathrm{mol} / \mathrm{l})$

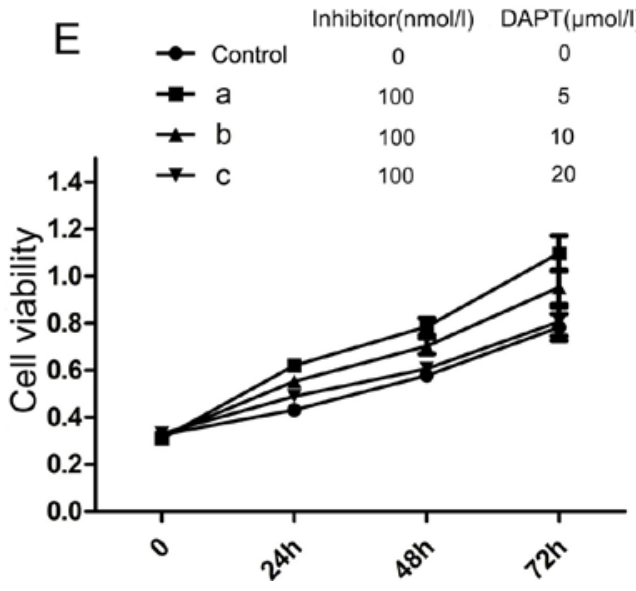

Figure 2. miR-125b inhibits the Notch signaling pathway and cell proliferation. (A) Following transfection of HaCaT for $48 \mathrm{~h}$ with the miR-125b inhibitor $(50 \mathrm{nmol} / \mathrm{l})$ or inhibitor $\mathrm{NC}(50 \mathrm{nmol} / \mathrm{l})$, the levels of miR- $125 \mathrm{~b}$ were detected by reverse transcription-quantitative polymerase chain reaction analysis to verify the transfection. (B) Following transfection of HaCaT cells for $48 \mathrm{~h}$ with the miR-125b inhibitor (100 nmol/l), the mRNA expression levels of the downstream genes of the Notch signaling pathway were detected by RT-qPCR. " $\mathrm{P}<0.05$ vs. control. (C) Following HaCaT cells being transfected for $48 \mathrm{~h}$ with the miR-125b inhibitor $(100 \mathrm{nmol} / \mathrm{l})$, the protein expression levels of the downstream gene of the Notch signaling pathway were detected using western blot analysis. (D) $\mathrm{HaCaT}$ cells were transfected and proliferated with three different concentrations of the miR-125b inhibitor. The viability of HaCaT cells was monitored using CCK-8 assays. (E) In the presence of the miR-125b inhibitor (100 nmol/l), three different concentrations of DAPT were added to the HaCaT cells. The viability of $\mathrm{HaCaT}$ cells was monitored using CCK-8 assays. The results are shown in the proliferation curve of the $\mathrm{HaCaT}$ cells. ${ }^{*} \mathrm{P}<0.05$, ${ }^{* * *} \mathrm{P}<0.001$. miR, microRNA; CCK-8, Cell Counting Kit-8.

UGC-3'. The sequence of si-NC was 5'-CCAUCUCCCGGU ACAAAAUCUGCU-3'. At $48 \mathrm{~h}$ following transfection, the cells were used for protein extraction.
Luciferase reporter assays. The wild-type 3'-UTR (3'-UTR-WT) of BRD4 containing the miR-125b binding sites was purchased from Guangzhou RiboBio Co., Ltd. Mutant 


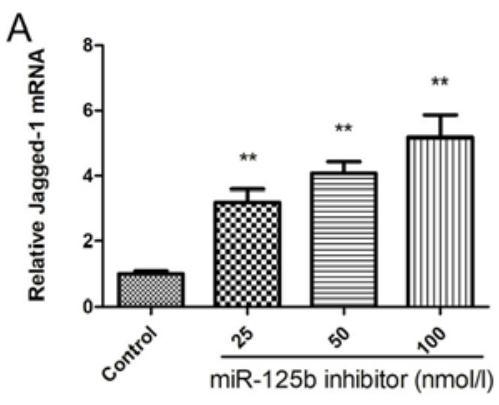

B

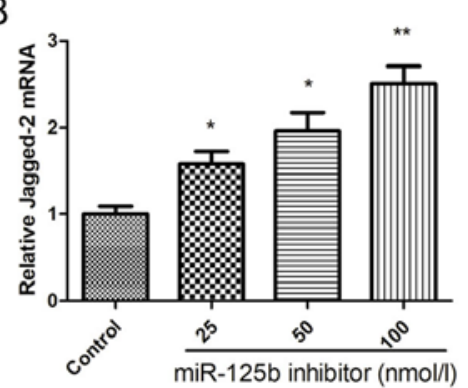

C

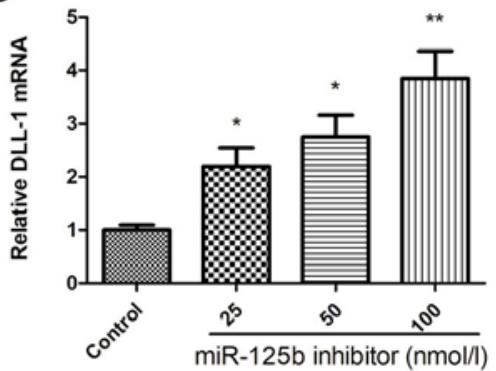

D

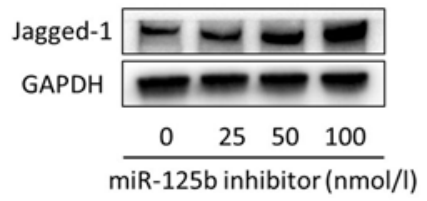

$\mathrm{E}$

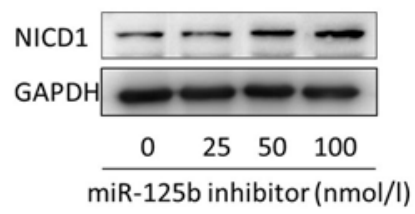

$\mathrm{F}$

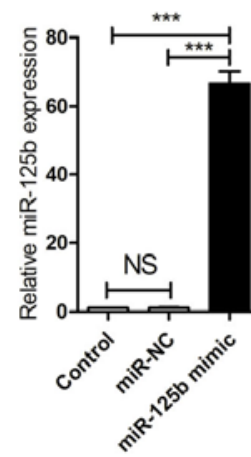

G

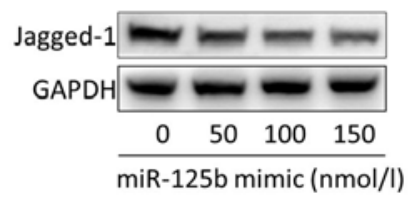

$\mathrm{J}$

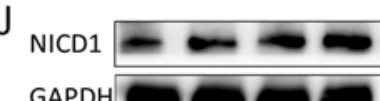

miR-125b mimic

(150nmol/l)

Jagged-1( $\mu \mathrm{g} / \mathrm{ml}) \quad 0 \quad 0.5 \quad 1 \quad 1.5$

Figure 3. miR-125b inhibits the activation of the Jagged1/Notch signaling pathway. At $48 \mathrm{~h}$ after the HaCaT cells being transfected with the three different concentrations of miR-125b inhibitor, (A) Jagged-1, (B) Jagged-2 and (C) DLL1 mRNA expression was detected by reverse transcription-quantitative polymerase chain reaction analysis. (D and E) HaCaT cells were transfected with three different concentrations of miR-125b inhibitor, and the expression of (D) Jagged-1 and (E) NICD1 was detected by western blotting. (F) Following transfection of HaCaT cells for $48 \mathrm{~h}$ with the miR-125b mimic (50 nmol/1) or miR-NC $(50 \mathrm{nmol} / \mathrm{l})$, the levels of the miR-125b were detected via reverse transcription-quantitative polymerase chain reaction analysis to verify the transfection. Following HaCaT cells being transfected with three different concentrations of miR-125b mimic, the expression of (G) Jagged-1 and (H) NICD1 was detected by western blotting. (I) Following three different concentrations of miR-125b inhibitor and DAPT being added to HaCaT cells, the expression of Jagged-1 and NICD1 was detected by western blotting. (J) Following miR-125b mimic and different concentrations of Jagged-1 being added to HaCaT cells, the expression of NICD1 was detected by western blotting. ${ }^{*} \mathrm{P}<0.05,{ }^{* *} \mathrm{P}<0.01$ vs. control; ${ }^{* * *} \mathrm{P}<0.001$. DLL1, $\delta$-like canonical Notch ligand $1 ;$ miR, microRNA; NICD1, Notch1 intracellular domain 1.

BRD4 3-'UTR (3'-UTR-MUT), in which mutations occur in the conserved binding sites for miR-125b, was generated using overlapping extension PCR. The fragment of BRD4 3'-UTR-WT and the mutant 3'-UTR fragments were inserted downstream of the Renilla luciferase gene in psiCHECK $^{\mathrm{TM}}-2$ vectors (Guangzhou RiboBio Co., Ltd.). The sequence of 3'-UTR-WT of BRD4 included the putative binding sites of miR-125b, whereas the sequence of 3'-UTR-MUT of BRD4 did not. Subsequently, the psiCHECK-2 vectors with 3'-UTR-WT or 3'-UTR-MUT regions of BRD4 were co-transfected with miR-125b mimic or mimic control using Lipofectamine 2000 into 293T cells. At 2 days following transfection, luciferase assays were performed according to the manufacturer's protocols (Guangzhou RiboBio Co., Ltd.) in triplicate. Renilla luciferase activity was normalized firefly luciferase activity for each well, yielding a gene expression ratio. Duplicate readings were averaged to generate a single ratio value per well. Values were then normalized to the average value of the control within the same cell line and experiment to yield the relative luciferase activity.

Statistical analysis. SPSS 19.0 statistical software (IBM Corp., Armonk, NY, USA) was used for all statistical analysis. Data are presented as the mean \pm standard deviation, and data were analyzed using one-way analysis of variance, followed by a Bonferroni's post hoc test for multiple comparisons. All experiments were independently repeated 3 times and $\mathrm{P}<0.05$ was considered to indicate a statistically significant difference.

\section{Results}

Analysis of miRNA in the serum. Certain miRNAs, including miR-125b, miR-200c, miR-200b, miR-19b, miR-152, miR-296-5p, miR-30d, miR-611, miR-491-3p, miR-23b, miR-95, miR-210, miR-224, miR-26a, miR-200a, miR-27b, miR-328 and miR-376a, are associated with the in vitro and in vivo differentiation of human keratinocytes and are hypothesized to serve a role in the proliferation of psoriatic keratinocytes. As detected by RT-qPCR in the sera of 32 patients with psoriasis, the miR-125b expression in the group of patients with psoriasis was more markedly downregulated than that in the control group of healthy volunteers (Fig. 1). The expression levels of miR-23b, miR-95, miR-210, miR-224, miR-26a, miR-200a, miR-27b, miR-328 and miR-376a were also upregulated to varying degrees (data not shown). 
A BRD4 $5^{\prime} \ldots$ CUCCUUUCAUUUAAG--CUCAGGGU...
miR-125b $3^{\prime} \quad$ AgUGUUCAAUCCCAGAGUCCCU

B

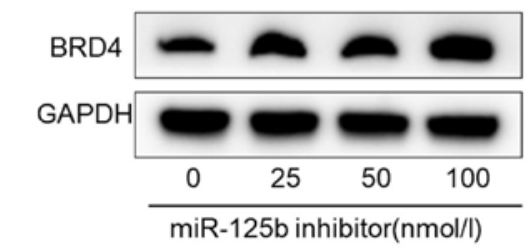

D

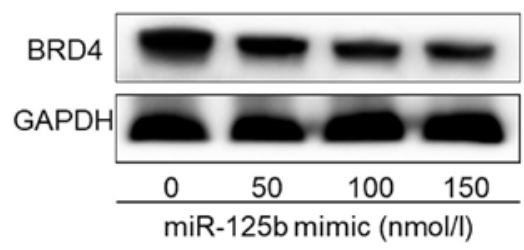

G

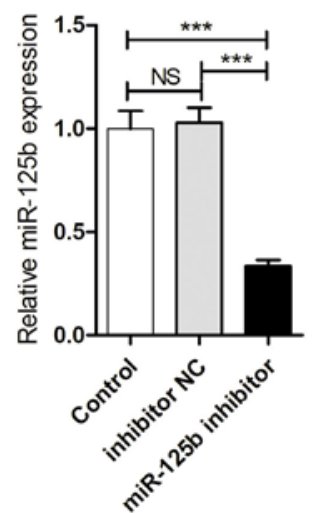

$\mathrm{H}$
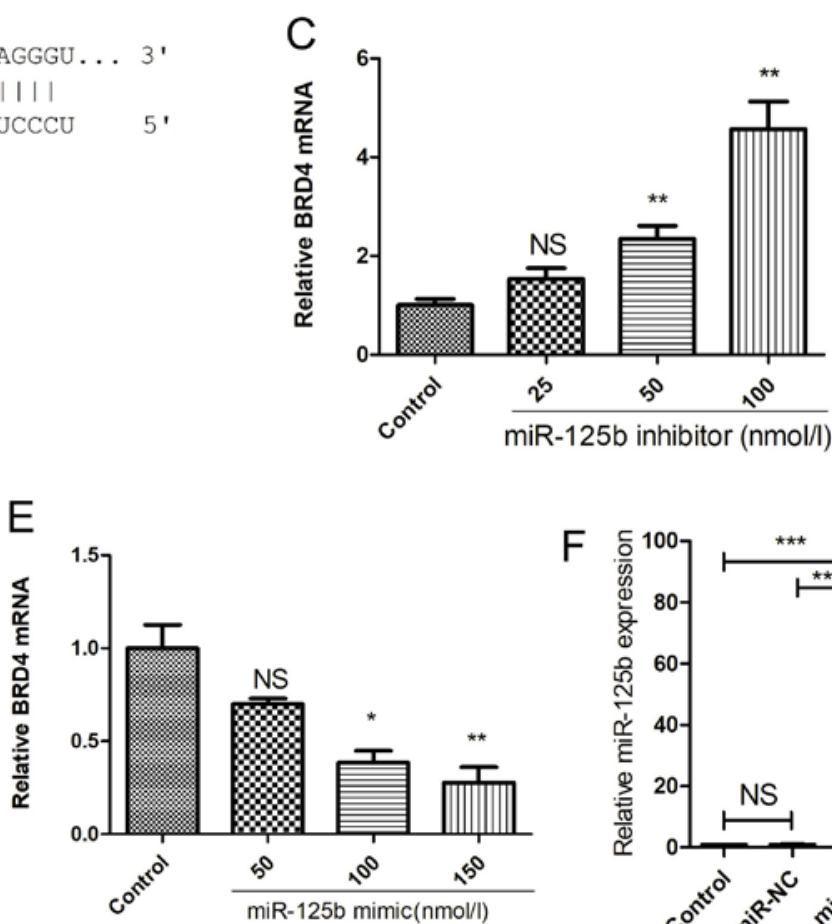

$\mathrm{F}$

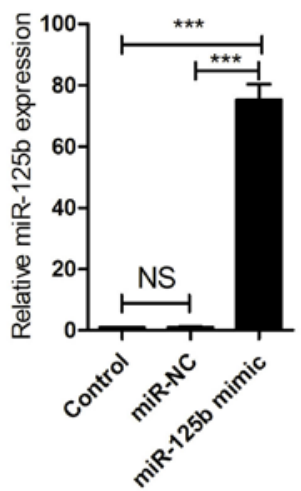

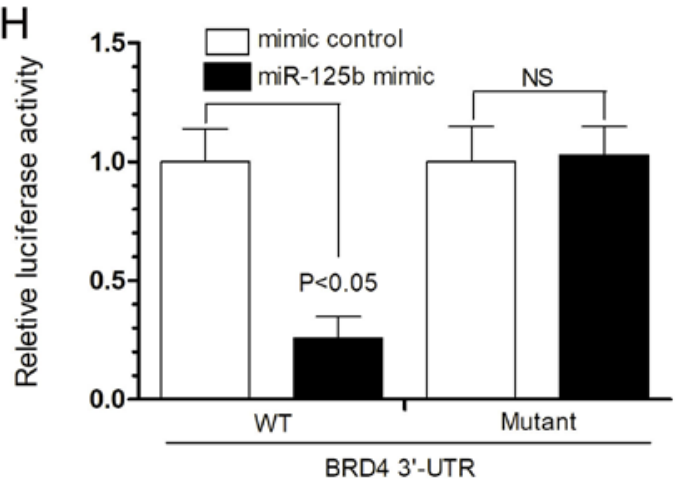

Figure 4. miR-125b binds to the BRD4 3'-UTR. (A) The binding site of miR-125b and the BRD4 3'-UTR was predicted. (B and C) At $48 \mathrm{~h}$ after HaCaT cells were transfected with the miR-125b inhibitor, the expression of BRD4 was detected by western blotting and RT-qPCR. (D and E) At $48 \mathrm{~h}$ after HaCaT cells were transfected with miR-125b mimic, the expression of BRD4 was detected by western blotting and RT-qPCR. Following transfection of 293T cells for $48 \mathrm{~h}$ with (F) miR-125b mimic $(50 \mathrm{nmol} / \mathrm{l})$ or miR-NC $(50 \mathrm{nmol} / \mathrm{l})$, or $(\mathrm{G}) \mathrm{miR}-125 \mathrm{~b}$ inhibitor $(50 \mathrm{nmol} / \mathrm{l})$ or inhibitor $\mathrm{NC}(50 \mathrm{nmol} / \mathrm{l})$, the levels of the miR-125b were detected by RT-qPCR to verify the transfection. (H) Reporter constructs containing either wild-type (WT) BRD4 3'-UTR, or BRD4 3'-UTR with mutation at the predicted miR-125b target sequence were co-transfected into $293 \mathrm{~T}$ cells, along with miR-125b mimic (50 $\mathrm{nmol} / \mathrm{l}) \mathrm{or}$ mimic control $(50 \mathrm{nmol} / 1)$. ${ }^{*} \mathrm{P}<0.05,{ }^{* *} \mathrm{P}<0.01$ vs. control; ${ }^{* * *} \mathrm{P}<0.001$; NS, not significant; BRD $\$$, bromodomain-containing protein 4; miR, microRNA; RT-qPCR, reverse transcription-quantitative polymerase chain reaction.

Inhibiting miR-125b upregulates the $m R N A$ and protein expression of downstream target genes of the Notch signaling pathway and increases the proliferation of HaCaT cells. Analysis of miRNA contents from the sera of patients with psoriasis revealed that miR-125b was downregulated. The abnormal proliferation of psoriatic keratinocytes is associated with the activation of the Notch signaling pathway. Therefore, the association between miR-125b and the Notch signaling pathway was investigated. miR-125b inhibitor was added to $\mathrm{HaCaT}$ cell culture fluid. Transfection with miR-125b inhibitor significantly decreased the expression of miR-125b in HaCaT cells (Fig. 2A). Additionally, after $48 \mathrm{~h}$, the mRNA and protein expression of downstream genes (Hesl, Heyl and Hey2) of the Notch signaling pathway was upregulated with markedly increased cell proliferation (Fig. 2B-D). The inhibitor DAPT of the Notch signaling pathway was added with the miR-125b inhibitor to the $\mathrm{HaCaT}$ cell culture fluid; consequently, $\mathrm{HaCaT}$ cell proliferation was inhibited (Fig. 2E).

Decreasing the abundance of miR-125b upregulates Jagged-1 expression and thereby activates the Notch signaling pathway. As indicated, the miR-125b inhibitor may upregulate the downstream genes of the Notch signaling pathway, and the cell proliferation mediated by the miR-125b inhibitor may be suppressed by an inhibitor of the Notch signaling pathway. Therefore, miR-125b may control HaCaT cell proliferation by regulating the Notch signaling pathway. Inhibiting miR-125b upregulated the expression of ligands of the Notch signaling pathway, including Jagged-1, Jagged-2 and $\delta$-like canonical Notch ligand 1 (DLL1; Fig. 3A-C). Jagged-1 is the most 

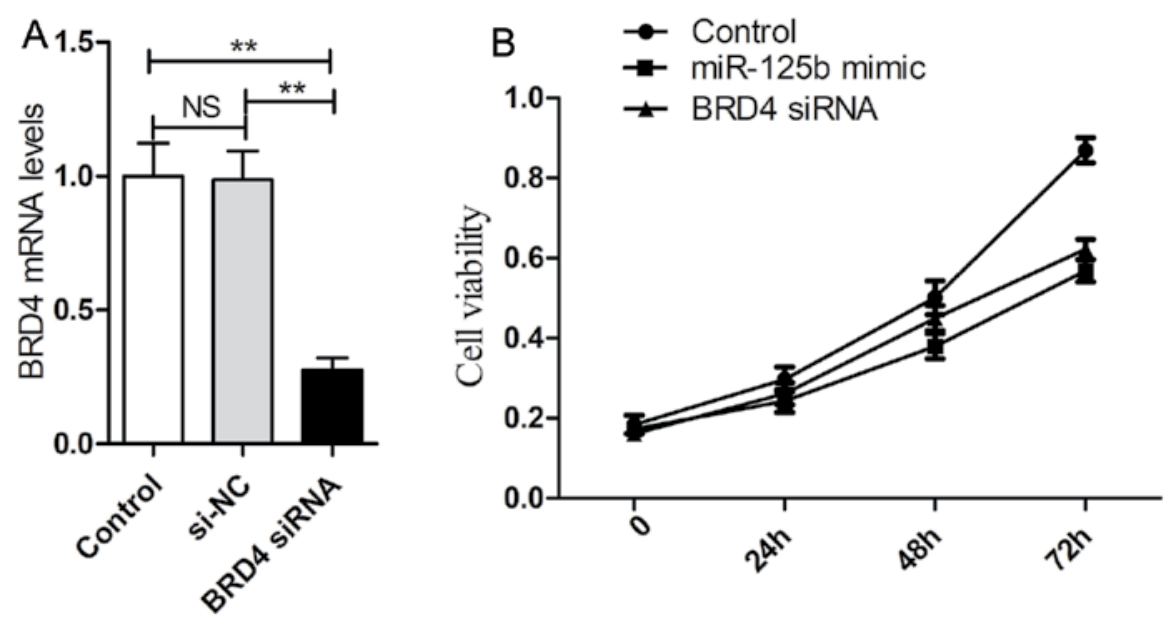

Figure 5. (A) Following transfection of HaCaT cells for $48 \mathrm{~h}$ with the BRD4 siRNA (50 nmol/l) or si-NC (50 nmol/l), the mRNA expression levels of the BRD4 were detected via reverse transcription-quantitative polymerase chain reaction analysis to verify the transfection. (B) BRD4 and miR-125b are associated with the proliferation of $\mathrm{HaCaT}$ cells. Following transfection of HaCaT cells with the BRD4 siRNA $(50 \mathrm{nmol} / \mathrm{l})$ or miR-125b mimic $(50 \mathrm{nmol} / \mathrm{l})$, the viability of $\mathrm{HaCaT}$ cells was determined using Cell Counting Kit-8 assays after transfect. ${ }^{* *} \mathrm{P}<0.01$. BRD4, bromodomain-containing protein 4; miR, microRNA; siRNA, small interfering RNA.

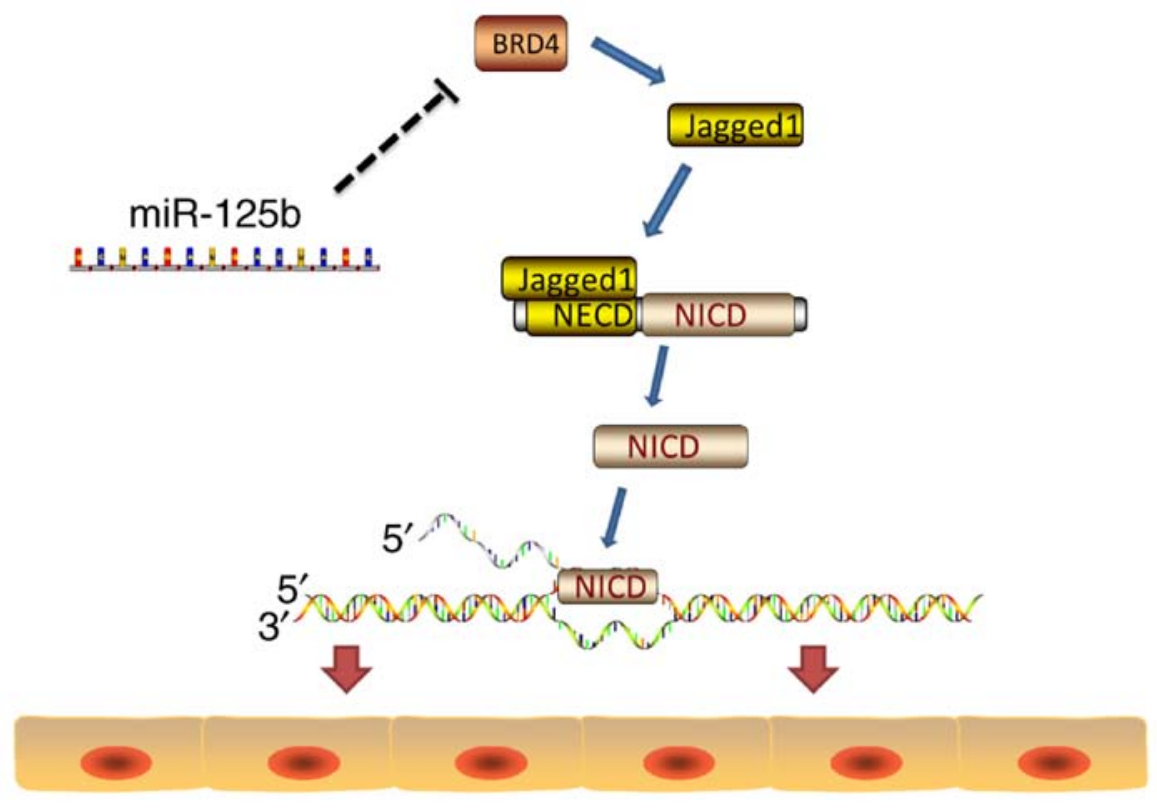

Figure 6. miR-125b inhibits the expression of the upstream protein BRD4 of the ligand Jagged-1 of the Notch signaling pathway and thereby inhibits proliferation in psoriasis. BRD4, bromodomain-containing protein 4; miR, microRNA; NECD, Notch extracellular domain; NICD, Notch1 intracellular domain.

markedly upregulated ligand among the five ligand types (Fig. 3A). Simultaneously, the Jagged-1 protein expression was increased by three different concentrations of miR-125b inhibitor (Fig. 3D). Furthermore, NICD1 is induced through miR-125b inhibitor (Fig. 3E). To further investigate the association between miR-125b and Notch signaling, cells were transfected with miR-125b mimic, which significantly upregulated miR-125b expression in HaCaT cells (Fig. 3F). When the miR-125b mimic was added, Jagged-1 was inhibited, and the release of active intracellular domain NICD1 from Notch1 was concurrently inhibited (Fig. 3G and H). Notch2 intracellular domain (NICD2) was upregulated, but to a lesser extent than NICD1 (data not shown). The miR-125b inhibitor and DAPT were then gradually added to the $\mathrm{HaCaT}$ cell culture fluid at increasing concentrations. As a result, Jagged-1 was upregulated, while NICD1 was downregulated. When DAPT was added to the inhibitor at the highest concentration, NICD1 expression was lowest (Fig. 3I), indicating that the Notch signaling pathway activation by miR-125b downregulation may be completely inhibited by DAPT, and miR-125b may suppress the activation of the Notch signaling pathway. When miR-125b mimic and soluble Jagged-1 fragment were added concomitantly, NICD1 was upregulated (Fig. 3J), implying that the Notch signaling pathway, as inhibited by miR-125b, may be activated by an ectogenic Jagged-1 fragment.

$m i R-125 b$ directly regulates the upstream protein BRD 4 of Jagged-1. miR-125b directly or indirectly regulates Jagged-1 
expression, thereby activating the Notch signaling pathway. By using two computational bioinformatics methods, namely TargetsScan and miRanda, it was verified that Jagged-1 was not a direct target of miR-125b, and that the BRD4 3'-UTR may include a direct binding site of miR-125b (Fig. 4A). To confirm the effect of miR-125b on the expression of BRD4, $\mathrm{HaCaT}$ cells were transfected with the miR-125b inhibitor for $48 \mathrm{~h}$. The mRNA and protein expression levels of BRD4 were markedly upregulated (Fig. 4B and C). By contrast, with the upregulated expression of miR-125b in HaCaT cells, the mRNA and protein expression levels of BRD4 were markedly decreased (Fig. 4D and E). Therefore, it was concluded that miR-125b inhibits BRD4.

To verify whether miR-125b directly acts on the 3'-UTR of BRD4, a luciferase assay was conducted in 293T cells transfected with miR-125b mimic or inhibitor (Fig. 4F and $G$ ). The luciferase activity was significantly decreased following co-transfection of BRD4-3'-UTR-WT and miR-125b. When BRD4-3'-UTR-Mutant and miR-125b were co-transfected, the inhibitory action of miR-125b was eliminated (Fig. 4H).

BRD4 siRNA and miR-125b mimic exhibit similar inhibitory effects on HaCaT cell proliferation. The effects of BRD4 and miR-125b on HaCaT cell proliferation were determined, and it was revealed that either the transfection of BRD4 siRNA or miR-125b mimic markedly reduced cell proliferation (Fig. 5).

\section{Discussion}

Psoriasis is a chronic inflammatory disease characterized by the abnormal differentiation and hyperproliferation of epidermal keratinocytes (3). It has several major histopathological changes, including hyperproliferation of keratinocytes, parakeratosis, inflammatory cell infiltration and neovascularization, as well as scaly erythema (3). At present, the etiology and pathogenesis of psoriatic skin lesions are too complex to be fully elucidated (19). The hyperproliferation of psoriatic epidermal cells is associated with the Notch signaling pathway (20). Furthermore, numerous miRNAs participate in the pathophysiological process of psoriasis $(21,22)$. In the present study, miR-125b potentially regulates the activation of the Notch signaling pathway by inhibiting the upstream protein BRD4 of Jagged-1 in psoriasis.

Previous studies $(14,15)$ have demonstrated that miR-125b is mainly associated with inflammation, cell proliferation and differentiation in psoriasis. miR-125b acts on ubiquitin-specific peptidase 2 to induce psoriasis (23). Injecting lipopolysaccharide may decrease miR-125b levels in mice, and the direct target gene of miR-125b is TNF- $\alpha$. Furthermore, decreased miR-125b levels may promote TNF- $\alpha$ secretion (24). miR-125b has also been demonstrated to be associated with hyperproliferation. Furthermore, miR-125b decreases keratinocyte proliferation by targeting fibroblast growth factor receptor-2 (14). HaCaT cells $(25,26)$ are a non-tumor-immortalized human normal skin keratinocyte strain characterized by continuous differentiation and proliferation characteristics similar to normal human keratinocytes, and are thus ideal as a cell model for studying psoriasis. In the present study, adding miR-125b inhibitor to
HaCaT cell culture fluid markedly increased proliferation. The Notch signaling pathway is associated with cell proliferation (27). Next, the regulatory effect of miR-125b on the proliferation of $\mathrm{HaCaT}$ cells via the Notch signaling pathway was investigated. Previous studies have demonstrated that Notch-1 is involved in the abnormal proliferation and differentiation of vascular endothelial cells $(28,29)$. In patients with psoriasis, the mRNA and protein expression levels of Notch-related proteins, including Notch-1, Notch-2, Jagged-1 and Hes1, were markedly decreased following treatment with biological agents. Therefore, the Notch signaling pathway is a key signaling pathway involved in the onset and development of psoriasis (30). In the Notch signaling pathway, the cells are the ligands/receptors to each other, and the ligand and receptor of the Notch signaling pathway are membrane proteins (31). Following ligand binding, the receptor is sheared by the $\gamma$-secretase in the transmembrane region. The active intracellular domain (NICD) is released into the nucleus, and the downstream pathway is activated (32). The Notch signaling pathway is specifically blocked by a $\gamma$-secretase inhibitor (33). To verify whether inhibiting miR-125b results in HaCaT cell proliferation, the inhibitor DAPT of the Notch signaling pathway was used along with the miR-125b inhibitor. HaCaT cell proliferation was inhibited, and miR-125b regulates such proliferation via the Notch signaling pathway.

Following miR-125b being inhibited, the mRNA and protein expression of downstream genes (Hesl, Heyl and Hey2) of the Notch signaling pathway were upregulated, indicating that the Notch signaling pathway was activated following miR-125b inhibition. In order to detect which site of the Notch signaling pathway was activated and in turn, which instigated the pathway, four types of receptors and five types of ligands of the Notch signaling pathway were investigated. The results demonstrated that the mRNA expression of any of the four types of Notch receptor was not upregulated (Notch-1, Notch-2, Notch-3 or Notch-4); however, mRNA expression was upregulated for three (Jagged-1, Jagged-2 and DLL1) of the five types of ligands, most notably Jagged-1. Therefore, Jagged-1 was selected as the research target. Following knockdown of endogenous miR-125b expression, Jagged-1 and NICD1 were upregulated. Of note, NICD2 was also upregulated; NICD1 possesses the structural fragment(s) of the intracellular domains of all the Notch receptors and is more highly expressed than NICD2. Therefore, the changes in NICD1 expression were highlighted. Transfection with miR-125b mimic revealed that Jagged-1 and NICD1 expression levels were downregulated, indicating that miR-125b directly or indirectly acts on the Notch signaling pathway.

The results of the present study demonstrated that BRD4 was a potential target gene of miR-125b; it was previously reported to be an upstream signal molecule of Jagged-1 (34). BET proteins, including BRDT, BRD2, BRD3 and BRD4, are novel 'readers' of epigenetic information. These proteins regulate the activity of transcription factors by binding to histone or non-histone lysine residues. Among these BET proteins, BRD4 is the most widely studied. In the NF- $\kappa \mathrm{B}$ signaling pathway associated with tumorigenesis, BRD4 recruits positive transcription elongation factor $b$ proteins that activate the $N F-\kappa B$ signaling pathway and are critical for nuclear 
signaling. The BRD4 and NF- $\mathrm{BB}$ enhancers also bind to each other and form a 'super enhancer' complex that stimulates the $\mathrm{NF}-\kappa \mathrm{B}$ signaling pathway. Luciferase assay results confirmed that miR-125b directly targets the BRD4 3'-UTR.

In summary, miR-125b tightly binds to 3'-UTR of BRD4 with highly-marching sequences and restrains the translation process of the Jagged-1 ligand. By further inhibiting the activation of the Notch signaling pathway, miR-125b suppresses the proliferation of psoriasis cells. Additionally, miR-125b is a critical molecule in the progress of psoriasis.

The limitations of the present study were as follows: The clinical sample size was small, and only few previous studies had investigated BRD4 through clinical samples. Given the extremely strong binding of BRD4 to histones and non-histone lysine residues, the mechanisms of actions of BRD4 on any of the ligands or receptors of the Notch signaling pathway have not been investigated. Previous studies have focused on miR-125b and the activation of T cells. Various activated T-cell subsets, including Thl, Th2, Treg, Thl7 and CD8 T, under the action of different adhesion factors, migrate to the skin and aggregate. Numerous cytokines are then simultaneously released to stimulate the hyperproliferation of keratinocytes, with an inflammatory reaction after the epidermis considerably thickens $(35,36)$. By investigating the differentiation/proliferation of psoriasis cells, the significance of the present study lies in its discovery that miR-125b does not directly act on the Notch signaling pathway but on the upstream molecule BRD4 of the ligand Jagged-1 of the Notch signaling pathway (Fig. 6). Therefore, the miRNA indirectly regulates the Notch signaling pathway. Furthermore, the Notch signaling pathway is associated with the onset of psoriasis. Therefore, the present study is of great importance in the regulation and treatment of psoriasis. miR-125b may be a potential biomarker and therapeutic target for the treatment of psoriasis.

The present study revealed that miR-125b inhibits the expression of the upstream protein BRD4, and of the ligand Jagged-1 of the Notch signaling pathway, thereby inhibiting the $\mathrm{HaCaT}$ cell proliferation that is associated with the activation of the Notch signaling pathway in psoriasis.

\section{Acknowledgements}

Not applicable.

\section{Funding}

No funding was received.

\section{Availability of data and materials}

The datasets used during the current study are available from the corresponding author on reasonable request.

\section{Authors' contributions}

MP and DL conceived and designed the study. YH and XZ designed and performed data analyses. MP and XL collected the data and drafted the manuscript. All authors read and approved the final manuscript.

\section{Ethics approval and consent to participate}

The study procedures were approved by the Ethics Committee of the Northern Jiangsu Province Hospital. Written informed consent was obtained from all subjects.

\section{Patient consent for publication}

Identifying information, including names, initials, date of birth or hospital numbers, images or statements were not included in this manuscript. The patients consented for the publication of the associated data.

\section{Competing interests}

The authors declare that they have no competing interests.

\section{References}

1. Smith RL, Warren RB, Griffiths CE and Worthington J: Genetic susceptibility to psoriasis: An emerging picture. Genome Med 1: 72, 2009.

2. Nestle FO, Kaplan DH and Barker J: Psoriasis. N Engl J Med 361: 496-509, 2009

3. Lowes MA, Bowcock AM and Krueger JG: Pathogenesis and therapy of psoriasis. Nature 445: 866-873, 2007.

4. Miele L: Notch signaling. Clin Cancer Res 12: 1074-1079, 2006.

5. Jiao Z, Wang W, Guo M, Zhang T, Chen L, Wang Y, You H and Li J: Expression analysis of Notch-related molecules in peripheral blood $\mathrm{T}$ helper cells of patients with rheumatoid arthritis. Scand J Rheumatol 39: 26-32, 2010.

6. Murea M, Park JK, Sharma S, Kato H, Gruenwald A, Niranjan T, Si H, Thomas DB, Pullman JM, Melamed ML and Susztak K: Expression of Notch pathway proteins correlates with albuminuria, glomerulosclerosis, and renal function. Kidney Int 78: 514-522, 2010.

7. Nickoloff BJ and Nestle FO: Recent insights into the immunopathogenesis of psoriasis provide new therapeutic opportunities. J Clin Invest 113: 1664-1675, 2004.

8. Lehar SM and Bevan MJ: Immunology: Polarizing a T-cell response. Nature 430: 150-151, 2004.

9. Napolitani G,Rinaldi A,Bertoni F, SallustoFand Lanzavecchia A: Selected Toll-like receptor agonist combinations synergistically trigger a $\mathrm{T}$ helper type 1-polarizing program in dendritic cells. Nat Immunol 6: 769-776, 2005.

10. Xia $\mathbf{J}$ and Zhang W: MicroRNAs in normal and psoriatic skin. Physiol Genomics 46: 113-122, 2014.

11. Yan S, Xu Z, Lou F, Zhang L, Ke F, Bai J, Liu Z, Liu J, Wang H, Zhu $\mathrm{H}$, et al: NF- $\mathrm{KB}$-induced microRNA-31 promotes epidermal hyperplasia by repressing protein phosphatase 6 in psoriasis. Nat Commun 6: 7652, 2015.

12. Meisgen F, Xu N, Wei T, Janson PC, Obad S, Broom O, Nagy N, Kauppinen S, Kemény L, Ståhle M, et al: MiR-21 is up-regulated in psoriasis and suppresses T cell apoptosis. Exp Dermatol 21: 312-314, 2012.

13. Zhang L, Stokes N, Polak L and Fuchs E: Specific microRNAs are preferentially expressed by skin stem cells to balance self-renewal and early lineage commitment. Cell Stem Cell 8: 294-308, 2011.

14. Xu N, Brodin P, Wei T, Meisgen F, Eidsmo L, Nagy N, Kemeny L, Ståhle M, Sonkoly E and Pivarcsi A: MiR-125b, a microRNA downregulated in psoriasis, modulates keratinocyte proliferation by targeting FGFR2. J Invest Dermatol 131: 1521-1529, 2011.

15. Huang RY, Li L, Wang MJ, Chen XM, Huang QC and Lu CJ: An exploration of the role of MicroRNAs in psoriasis: A systematic review of the literature. Medicine (Baltimore) 94: e2030, 2015.

16. Andrieu GP, Shafran JS, Deeney JT, Bharadwaj KR, Rangarajan A and Denis GV: BET proteins in abnormal metabolism, inflammation, and the breast cancer microenvironment. J Leukoc Biol 104: 265-274, 2018. 
17. Zhang G, Liu R, Zhong Y, Plotnikov AN, Zhang W, Zeng L, Rusinova E, Gerona-Nevarro G, Moshkina N, Joshua J, et al: Down-regulation of $\mathrm{NF}-\kappa \mathrm{B}$ transcriptional activity in HIV-associated kidney disease by BRD4 inhibition. J Biol Chem 287: 28840-28851, 2012.

18. Livak KJ and Schmittgen TD: Analysis of relative gene expression data using real-time quantitative PCR and the 2(-Delta Delta C(T)) method. Methods 25: 402-408, 2001.

19. Crow JM: Therapeutics: Silencing psoriasis. Nature 492: S58-S59, 2012.

20. Ota T, Takekoshi S, Takagi T, Kitatani K, Toriumi K, Kojima T, Kato M, Ikoma N, Mabuchi T and Ozawa A: Notch signaling may be involved in the abnormal differentiation of epidermal keratinocytes in psoriasis. Acta Histochem Cytochem 47: 175-183, 2014

21. Jinnin M: Recent progress in studies of miRNA and skin diseases. J Dermatol 42: 551-558, 2015.

22. Liu Q, Wu DH, Han L, Deng JW, Zhou L, He R, Lu CJ and Mi QS: Roles of microRNAs in psoriasis: Immunological functions and potential biomarkers. Exp Dermatol 26: 359-367, 2017.

23. Wei T, Folkersen L, Biskup E, Xu N, Manfe V, Niazi O and Gniadecki R: Ubiquitin-specific peptidase 2 as a potential link between microRNA-125b and psoriasis. Br J Dermatol 176 : 723-731, 2017.

24. Tili E, Michaille JJ, Cimino A, Costinean S, Dumitru CD, Adair B, Fabbri M, Alder H, Liu CG, Calin GA and Croce CM: Modulation of miR-155 and miR-125b levels following lipopolysaccharide/TNF-alpha stimulation and their possible roles in regulating the response to endotoxin shock. J Immunol 179: 5082-5089, 2007.

25. Boukamp P, Petrussevska RT, Breitkreutz D, Hornung J, Markham A and Fusenig NE: Normal keratinization in a spontaneously immortalized aneuploid human keratinocyte cell line. J Cell Biol 106: 761-771, 1988.

26. Schürer N, Köhne A, Schliep V, Barlag K and Goerz G: Lipid composition and synthesis of HaCaT cells, an immortalized human keratinocyte line, in comparison with normal human adult keratinocytes. Exp Dermatol 2: 179-185, 1993.
27. Thélu J, Rossio P and Favier B: Notch signalling is linked to epidermal cell differentiation level in basal cell carcinoma, psoriasis and wound healing. BMC Dermatol 2: 7, 2002.

28. Abdou AG, Maraee AH, Sharaf A and Elnaidany NF: Up-regulation of Notch-1 in psoriasis: An immunohistochemical study. Ann Diagn Pathol 16: 177-184, 2012.

29. Rooney P, Connolly M, Gao W, McCormick J, Biniecka M, Sullivan O, Kirby B, Sweeney C, Molloy E, Markham T, et al: Notch-1 mediates endothelial cell activation and invasion in psoriasis. Exp Dermatol 23: 113-118, 2014.

30. Skarmoutsou E, Trovato C, Granata M, Rossi GA, Mosca A, Longo V, Gangemi P, Pettinato M, D'Amico F and Mazzarino MC: Biological therapy induces expression changes in Notch pathway in psoriasis. Arch Dermatol Res 307: 863-873, 2015.

31. Kovall RA, Gebelein B, Sprinzak D and Kopan R: The canonical Notch signaling pathway: Structural and biochemical insights into shape, sugar, and force. Dev Cell 41: 228-241, 2017.

32. Bray SJ and Gomez-Lamarca M: Notch after cleavage. Curr Opin Cell Biol 51: 103-109, 2018.

33. Locatelli M and Curigliano G: Notch inhibitors and their role in the treatment of triple negative breast cancer: Promises and failures. Curr Opin Oncol 29: 411-427, 2017.

34. Andrieu G, Tran AH, Strissel KJ and Denis GV: BRD4 regulates breast cancer dissemination through Jagged1/Notch1 signaling. Cancer Res 76: 6555-6567, 2016.

35. Diani M, Altomare G and Reali E: T cell responses in psoriasis and psoriatic arthritis. Autoimmun Rev 14: 286-292, 2015.

36. Chen H, Liu H, Lu C, Wang M, Li X, Zhao H, Yan Y, Yu W, Han L and Dai Z: PSORI-CM02 formula increases CD4+ Foxp3+ regulatory $\mathrm{T}$ cell frequency and ameliorates imiquimod-induced psoriasis in mice. Front Immunol 8: 1767, 2018.

This work is licensed under a Creative Commons Attribution-NonCommercial-NoDerivatives 4.0 International (CC BY-NC-ND 4.0) License. 